\title{
EVALUATION OF PERFUSION AND THERMAL PARAMETERS OF SKIN TISSUE USING COLD PROVOCATION AND THERMOGRAPHIC MEASUREMENTS
}

\author{
Maria Strąkowska ${ }^{1)}$, Robert Strąkowski ${ }^{1)}$, Michał Strzelecki' ${ }^{1)}$, Gilbert de Mey ${ }^{2)}$, \\ Bogusław Więcek ${ }^{1)}$
}

1) Lodz University of Technology, Faculty of Electrical, Electronic, Computer and Control Engineering, Wólczańska 211/215, 90-924 Łódź, Poland(strakowska.m@gmail.com,robert.strakowski@p.lodz.pl, \michal.strzelecki@p.lodz.pl, +48 42 631 2631,wiecek@p.lodz.pl)

2) University of Gent, Department of Electronics and Information Systems, B-9000 Gent, Sint Pieternieuwstraat 41, Belgium (gdemey@ugent.be)

\begin{abstract}
Measurement of the perfusion coefficient and thermal parameters of skin tissue using dynamic thermography is presented in this paper. A novel approach based on cold provocation and thermal modelling of skin tissue is presented. The measurement was performed on a person's forearm using a special cooling device equipped with the Peltier module. The proposed method first cools the skin, and then measures the changes of its temperature matching the measurement results with a heat transfer model to estimate the skin perfusion and other thermal parameters. In order to assess correctness of the proposed approach, the uncertainty analysis was performed.
\end{abstract}

Keywords: active thermovision, perfusion, thermal model, skin tissue, measurement uncertainty.

C) 2016 Polish Academy of Sciences. All rights reserved

\section{Introduction}

Thermal modelling of a tissue is still a challenging object of research. One of the first heat transfer models of skin was developed by Pennes more than half a century ago [1]. This model describes the skin perfusion in a simplified form. Many authors are still using this model in their new research despite of being frequently criticized, mainly because the heat transfer by perfusion is modelled taking into account the bloodstream temperature difference [2]. Other authors proposed a thermal analysis based on heat transfer between the tissue and a large vessel $[3,4]$. A progress in thermal modelling took place when it was noticed that there was a significant heat transfer in the larger blood vessels of a diameter of 50-150 $\mu \mathrm{m}$ [2]. As a result, the thermal model of skin was divided into 2 parts, one for the tissue and the second for the arteries [2]. The so-called effective thermal parameters of tissue were defined depending on the ratio of tissue and artery volumes. An important step forward was made by finding that the heat exchange between small arteries and veins typically occurs in parallel [5-7]. Next, the thermoregulatory mechanism was added to the thermal simulations of tissues [8]. Nowadays, there are plenty of new analytical and numerical thermal analyses of the human tissues [9-19]. Moreover, the inverse thermal problem simulations are applied to predict the values of tissue parameters [13-15]. Most of the models were developed for steady state conditions, but recently some new time-dependent approaches have been published [13-16]. Nowadays, there is a need of dynamic thermal analysis of the human tissues mainly due to the new applications of active thermography in medicine using cold provocation for screening $[14,17]$.

In this research we use the inverse thermal modelling to evaluate the perfusion defined according to the Pennes model, as well as the thermal parameters of multilayer tissue structure 
of skin (i.e. its thermal conductivity and capacity). In this approach we use modelling in the Laplace domain and optimization based on matching the Nyquist's plots of the thermal impedance obtained from the model and the experiment [13-15]. In addition, the uncertainty analysis was also performed to confirm practical usefulness of the proposed method. We intend to apply this approach for medical screening of e.g. malignant and benign skin tumours.

\section{Multilayer thermal model of tissue}

Heat transfer in human tissue can be modelled using the Pennes equation [1]. In this paper, the skin is assumed to be a 3-layer structure consisting of epidermis, dermis and hypodermis. The proposed method is based on cold provocation of the skin and measuring - by an infrared camera - its temperature changes over a period of time $[14,15]$. After introductory cooling, the skin temperature is returning to the normal equilibrium state. Typically, due to the large thermal mass, this process can take a few tens of minutes.

In the thermal model it is assumed that the heat transfer through the skin to the ambience is one-dimensional (1). Also, the heat flux and temperature at the interface between the layers are assumed to be continuous (7-9). For each layer of skin the perfusion coefficient $w_{i}(1 / \mathrm{s})$, thermal conductivity $k_{i}(\mathrm{~W} /(\mathrm{m} \cdot \mathrm{K}))$, specific heat per unit weight $c_{i}(\mathrm{~J} /(\mathrm{kg} \cdot \mathrm{K}))$, density $\rho_{i}$ $\left(\mathrm{kg} / \mathrm{m}^{3}\right)$ and thickness $d_{i}(\mathrm{~m})$ are concerned. The temperature in the $i$-th layer is described by using the energy balance (1):

$$
\rho_{i} \cdot c_{i} \cdot \frac{d T_{i}}{d t}=k_{i} \cdot \frac{d^{2} T_{i}}{d x^{2}}+w_{i} \cdot c_{b} \cdot \rho_{b}\left(T_{B}-T_{i}\right),
$$

where: $c_{b}$ denotes the specific heat of blood per unit weight; $\rho_{b}$ - the blood density and $T_{B}-$ the blood temperature assumed as a reference temperature equal to $0^{\circ} \mathrm{C}$.

The equation (1) can be easily transformed onto the frequency domain (2-4):

$$
\begin{gathered}
k_{i} \frac{d^{2} T_{i}}{d x^{2}}=\rho_{i} \cdot c_{i} \cdot s \cdot T_{i}+w_{i} \cdot c_{b} \cdot \rho_{b} \cdot T_{i}, \\
k_{i} \frac{d^{2} T_{i}}{d x^{2}}-T_{i}\left(\rho_{i} \cdot c_{i} \cdot s \cdot T_{i}+w_{i} \cdot c_{b} \cdot \rho_{b} \cdot T_{i}\right)=0, \\
\frac{d^{2} T_{i}}{d x^{2}}-\frac{T_{i}}{L_{i}^{2}}=0,
\end{gathered}
$$

where $L$ is the complex number interpreted as a diffusion length (5):

$$
L_{i}(s)=\frac{1}{\sqrt{\left(\rho_{i} \cdot c_{i} \cdot s+w_{i} \cdot c_{b} \cdot \rho_{b}\right) / k_{i}}} .
$$

The equation (4) has the analytical solution for each $i$-th layer (6):

$$
T_{i}(s)=A_{i}(s) \cdot e^{\frac{x}{L_{i}(s)}}+B_{i}(s) \cdot e^{\frac{x}{L_{i}(s)}} .
$$

Using the boundary and initial conditions given by (7-9), the integration variables $A_{i}$ and $B_{i}$ can be found:

$$
\begin{gathered}
\left.k_{1} \frac{d T_{1}}{d x}\right|_{x=0}=\left.h \cdot T_{1}\right|_{x=0}+P_{A C},\left.\quad k_{i} \frac{d T_{i}}{d x}\right|_{x=d_{i}}=\left.k_{i+1} \frac{d T_{i+1}}{d x}\right|_{x=0}, \\
\left.T_{i}\right|_{x=d_{i}}=\left.T_{i+1}\right|_{x=0}, \\
\left.T_{3}\right|_{x=d_{3}}=T_{B} .
\end{gathered}
$$




\section{Methodology of measuring skin parameters}

\subsection{Thermographic measurement}

The measurements were performed using a Cedip Titanium InSb $640 \times 512$ MWIR camera. A part of forearm's skin was cooled by a few degrees with a cooling device equipped with the Peltier module (Fig. 1).

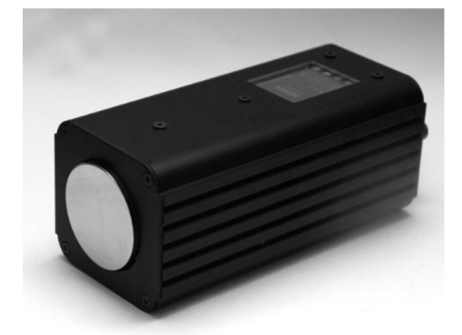

Fig. 1. A cooling device with the Peltier module.

The device is a portable cooler capable of setting a required temperature of the polished metal block within the range of $15-40^{\circ} \mathrm{C}$. It enables cooling or heating the skin. Using this device, different levels of thermal excitation can be chosen. The size and shape of active block were designed to uniformly cool or heat the skin surface to a required temperature. In this research, the temperature of metal block was set to $26^{\circ} \mathrm{C}$ and the device had been in contact with the skin for about 4 seconds. The camera had acquired the sequence of thermal images with the frame rate of $50 \mathrm{~Hz}$ for $10 \mathrm{~min}$. (30000 frames), till the skin reached its initial temperature. A few frames from the recorded sequence of images are shown in Fig. 2.

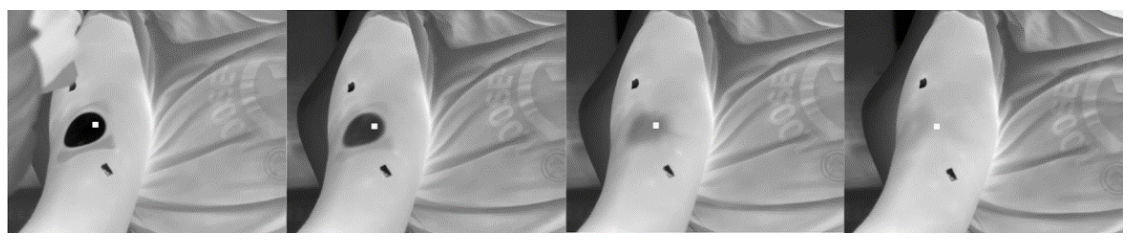

Fig. 2. The thermal sequence of images after cooling the skin, frames no. 140, 650, 4650, 20650.

The mean temperature of a given $9 \times 9$ pixel area was calculated for every frame of the sequence and then used to draw the curve of temperature rise over a period of time. Because the measurement had to be performed for the same Region of Interest (ROI) for each frame, a person's motion was compensated by software means. The movement correction method based on cross-correlation has been already presented in [20]. A sample of reference ROI for the movement correction algorithm is shown in Fig. 3.

In order to calculate the uncertainties corresponding to the estimated skin parameter values, two experiments were performed. First, the temperature rise in a function of time was measured for 10 small areas $(9 \times 9$ pixels $)$ situated close to each other. The chosen distance between the areas was equal to 8 pixels. Moreover, the selected areas were far away from the big vessels that were clearly visible in the thermographic images. These measurements were used to calculate the A-type component of uncertainty $u_{A}$. 


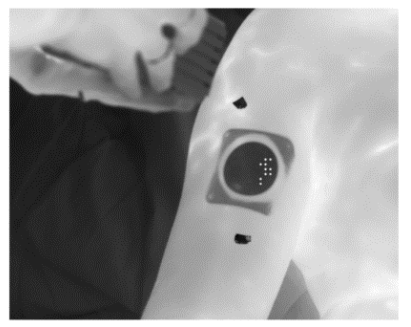

Fig. 3. The centres of areas used for further analysis marked in the first image of the sequence.

In the second experiment, a uniform noise was added to a selected measurement. It was repeated 12 times. The noise amplitude was $20 \mathrm{mK}$, that corresponds to the noise of the camera given by NETD (Noise Equivalent Temperature Difference). This procedure was used to calculate the uncertainty of temperature measurement caused by the thermographic camera - the B-type component of uncertainty $u_{B}$.

\subsection{Analysis in time domain}

The temperature rise values obtained over a period of time were approximated by a curve composed of the exponential and error functions (10). Examples of matching the measured and calculated curves in the linear and logarithmic scales are shown in Fig. 4:

$$
f(t)=A\left(1-e^{-\omega_{0} t}\right)+B\left(1-e^{\omega_{1} t} \cdot \operatorname{erfc}\left(\sqrt{\omega_{1}} \sqrt{t}\right)\right),
$$

where the coefficients $A, B$ correspond to contributions of the exponential $(A)$ and error function $(B)$ parts of the approximation.

a)

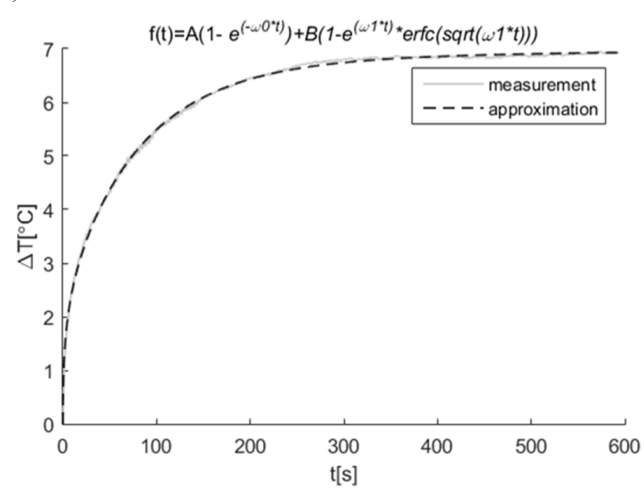

b)

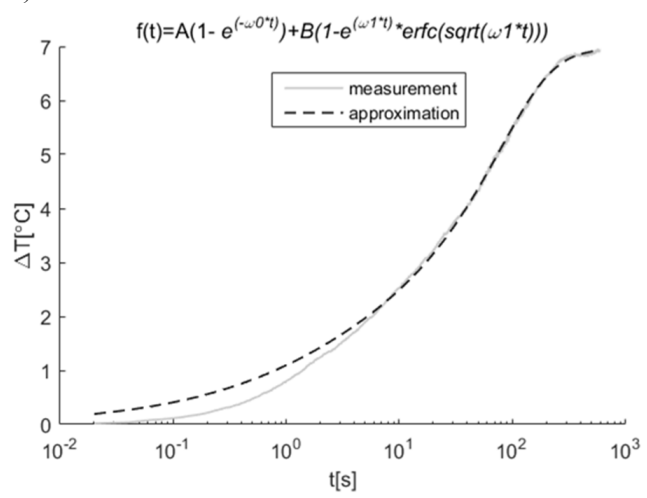

Fig. 4. The temperature rise in a function of time and its approximation, in a) linear and b) logarithmic time scales.

Approximation using the function (10) origins from the developed thermal model and matches well the thermographic measurement results after cold provocation [13, 14]. The matching using (10) is very good for the time greater than 6-7 s, but is slightly worse at the beginning of the thermal process. This problem will be considered in the future research. At this stage of development of the method the non-perfect approximation of the measurement curve for smaller time values is neglected. It should be noticed that the mismatching the curves in the first seconds of the process is at the level of camera accuracy, i.e. $0.2^{\circ} \mathrm{C}$. Moreover, 
the skin reaction after cold provocation takes about $20 \mathrm{~min}$. Therefore, matching the later part of the measurement curve is much more important.

\subsection{Analysis in frequency domain}

In order to find the values of perfusion coefficient as well as other thermal parameters of skin tissue, transformation from the time onto the frequency domain was accomplished. The function (10) after Laplace transformation takes a form (11):

$$
Z=\frac{A}{1+j \omega / \omega_{0}}+\frac{B}{1+\sqrt{j \omega / \omega_{1}}}
$$

where the coefficients $A, B$ have the same meanings as in (10).

Matching the Nyquist's plots obtained from the measurements and those from the model enabled to obtain the thermal parameters of tissue:

- thermal conductivity;

- thermal capacity;

- perfusion coefficient.

Optimization using the Pattern Search method was performed for estimation of the skin parameter values. This method was chosen because it did not use the gradients of the objective function and enables to optimize with bounds. The Pattern Search optimization is based on two steps: the so called exploratory and pattern moves. The first step improves the directions of movements, whereas the second one lengthens the moves as long as the improvement continues [21, 22]. The values of a few parameters were set and kept unchanged during the whole optimization procedure: the values of thicknesses, specific heat per unit weight, density of blood, and convective heat transfer coefficient. The values of these parameters were taken from the literature and they were equal to $d_{1}=0.00008 \mathrm{~m}, d_{2}=0.001 \mathrm{~m}, d_{3}=0.003 \mathrm{~m}, c_{b}$ $=3770 \mathrm{~J} /(\mathrm{kg} \cdot \mathrm{K}), \rho_{b}=1060 \mathrm{~kg} / \mathrm{m}^{3}, h=50 \mathrm{~W} /\left(\mathrm{m}^{2} \cdot \mathrm{K}\right)[23,24]$. The optimization procedure for the developed thermal model is numerically complex and time-consuming. Due to this fact the optimization was performed in a few stages. First, the thermal conductivity of each layer was estimated together with the power $\left(P_{A C}\right)$, then the density and the specific heat were evaluated, and finally the perfusion coefficient was calculated. It was assumed that the perfusion coefficients in dermis and hypodermis layers were equal. The procedure of optimization was repeated till the Nyquist's plots obtained from the model matched those from the measurement [13].

The optimization parameters for each stage were chosen manually. The initial values of the parameters in the first iteration were selected according to the literature, while their ranges were chosen experimentally. A narrower range leads to faster optimization.

\section{Uncertainty analysis}

The uncertainty of measurement results was calculated according to the specification presented in the literature $[25,26]$. In this research we present the uncertainty analysis for the perfusion, thermal conductivity and thermal capacity. The perfusion in the first tissue layer (epidermis) is neglected. It is assumed that the same perfusion is in the second (dermis) and third (hypodermis) layers. These assumptions agree with those given in the literature [23, 24]. For the thermal conductivity and capacity the analysis was performed independently for all three layers of skin tissue.

In order to estimate the uncertainty $u_{A}$, the measurements were repeated 10 times for 10 neighbouring areas of the skin. The mean values as well as the corresponding standard deviations of perfusion, thermal conductivity and capacity were calculated (12): 


$$
u_{A}(p)=s(\bar{p})=\sqrt{\frac{\sum_{i=1}^{N}\left(p_{i}-\bar{p}\right)^{2}}{N(N-1)}},
$$

where: $p$ is the given parameter (perfusion, thermal conductivity or thermal capacity); $\bar{p}$ is its mean value, while $N$ represents the number of measurements.

The results are presented in Table 1.

Table 1 . The uncertainty $u_{a}$ for analysed parameters of the skin tissue.

\begin{tabular}{|ccc|c|cc|}
\hline \multicolumn{3}{|c|}{ Parameter } & Mean value & \multicolumn{3}{|c|}{ Standard deviation } \\
\hline$k_{1}$ & (epidermis) & $(\mathrm{W} /(\mathrm{m} \cdot \mathrm{K}))$ & 0.100 & \multicolumn{2}{|c|}{$<0.001$} \\
\hline$k_{2}$ & (dermis) & $(\mathrm{W} /(\mathrm{m} \cdot \mathrm{K}))$ & 0.168 & 0.006 & $(10 \%)$ \\
\hline$k_{3}$ & (hypodermis) & $(\mathrm{W} /(\mathrm{m} \cdot \mathrm{K}))$ & 0.166 & 0.006 & $(12 \%)$ \\
\hline$C_{t h 1}$ & $\left(\mathrm{~J} /\left(\mathrm{m}^{3} \cdot \mathrm{K}\right)\right)$ & 3436488 & 115255 & $(3 \%)$ \\
\hline$C_{t h 2}$ & $\left(\mathrm{~J} /\left(\mathrm{m}^{3} \cdot \mathrm{K}\right)\right)$ & 4537494 & 137952 & $(3 \%)$ \\
\hline$C_{t h 3}$ & $\left(\mathrm{~J} /\left(\mathrm{m}^{3} \cdot \mathrm{K}\right)\right)$ & 5485882 & 110716 & $(2 \%)$ \\
\hline \multicolumn{2}{|c|}{} & $(1 / \mathrm{s})$ & 0.00240 & 0.00017 & $(6.5 \%)$ \\
\hline
\end{tabular}

It was more difficult to evaluate the uncertainty $u_{B}$ due to the indirect way of using the temperature measurements from the IR camera to estimate accuracy of the perfusion and thermal parameters. For this purpose the inverse thermal problem solution was employed, and therefore there was no direct relation between the considered quantities and the measured temperature.

In the measurement, only the temperature difference was used. Each value of temperature measured by the camera was decreased by the value obtained from the first measurement. As a consequence, the temperature evolution over a period of time always started from zero. It means that the uncertainty of measurement can be reduced due to the drift of the thermal camera. It should be bore in mind that the measurement took a couple of minutes and the camera took the cooled photos. In addition, the front part of the case of the camera was thermally stabilized. Therefore, it was assumed that the source of the uncertainty of measurement caused by the IR equipment was due to the value of NETD given by the manufacturer. The noise of camera was assumed to be at the level of $\Delta T= \pm 20 \mathrm{mK}$.

In this research, there was considered the uniform (rectangle) probability distribution of noise. The procedure of evaluation of the uncertainty $u_{B}$ consisted in adding the noise with such distribution and the span of $\pm 20 \mathrm{mK}$ to all temperature samples during recording the thermal images by the camera. This process was repeated 12 times followed by calculating the standard deviations of the perfusion and thermal parameters in order to estimate the value of $u_{B}$. It was assumed that distribution of the output quantities (perfusion and thermal parameters: thermal conductivity and thermal capacity, $C_{t h i}=\rho_{i} \cdot c_{i}$ ) as well as of the input data (temperature) was uniform. For this reason the scaling factor $1 / \sqrt{3}$ to obtain the final value of $u_{B}$ was used $[25,26]$. The final results of the uncertainty analysis are presented in Table 2. After calculating the combined uncertainty $u_{c}$, to obtain the extended uncertainty $U$ the coverage factor $k_{p}=2$ was used [26]. 
Table 2. The final results of the uncertainty analysis.

\begin{tabular}{|ccc|c|c|cc|}
\hline \multicolumn{3}{|c}{ Parameter } & $u_{B}$ & $u_{c}$ & \multicolumn{2}{c|}{$U=2^{*} u_{c}$} \\
\hline$k_{1}$ & (epidermis) & $(\mathrm{W} /(\mathrm{m} \cdot \mathrm{K}))$ & 0.000624 & 0.0006 & 0.001 & $(1 \%)$ \\
\hline$k_{2}$ & (dermis) & $(\mathrm{W} /(\mathrm{m} \cdot \mathrm{K}))$ & 0.011464 & 0.012 & 0.025 & $(15 \%)$ \\
\hline$k_{3}$ & (hypodermis) & $(\mathrm{W} /(\mathrm{m} \cdot \mathrm{K}))$ & 0.01157 & 0.013 & 0.026 & $(16 \%)$ \\
\hline$C_{t h 1}$ & $\left(\mathrm{~J} /\left(\mathrm{m}^{3} \cdot \mathrm{K}\right)\right)$ & 434890 & 449924 & 899848 & $(26 \%)$ \\
\hline$C_{t h 2}$ & $\left(\mathrm{~J} /\left(\mathrm{m}^{3} \cdot \mathrm{K}\right)\right)$ & 378971 & 403333 & 806666 & $(17 \%)$ \\
\hline$C_{t h 3}$ & $\left(\mathrm{~J} /\left(\mathrm{m}^{3} \cdot \mathrm{K}\right)\right)$ & 594073 & 604316 & 1208632 & $(22 \%)$ \\
\hline \multicolumn{2}{|c|}{$w$ (dermis and hypodermis) } & $(1 / \mathrm{s})$ & 0.0003 & 0.0003 & 0.0006 & $(27 \%)$ \\
\hline
\end{tabular}

The obtained values of relative uncertainty for measurement of perfusion and thermal parameters of tissue were at a level of $15-27 \%$. The higher uncertainty was observed for thermal capacities (below 30\%). In our opinion it was a quite satisfactory result. The tissue is a complex structure and its thermal parameters and perfusion depend on many factors, both internal (thermoregulation, temperature, viscosity, ...) and external (environmental conditions). In addition, the thermoregulation can influence the perfusion and thermal parameters of tissue. Therefore, the obtained uncertainty of skin parameter values can be regarded as fully acceptable.

\section{Conclusions}

In this research we present a new method for evaluation of perfusion, thermal conductivity and capacity of skin tissue. The method is based on the inverse thermal modelling and temperature measurement by a thermographic camera after cooling of skin. The thermal process was dynamic and lasted several minutes. Estimation of the skin parameters may in the future improve the classic diagnostic thermal imaging used in detection of early stages of diseases, e.g. the breast cancer [27].

The values of skin thermal parameters (thermal conductivity and capacity) obtained with the developed method are contained within the ranges reported in the literature. However, the thermal parameters of skin are not well defined. It should be bore in mind that skin is a kind of porous material with varying and strongly nonlinear parameters. The ranges of variation of these parameters are large, and depend very much on the physiological and pathological state of the skin. In this study the skin parameters were estimated for the forearm region only. In the future we are planning to extend our research onto other parts of the body. Also, influence of a patient's fatigue on the estimated perfusion values will be analysed.

The presented technique should be validated using other approaches. However, the perfusion factor obtained by the presented method cannot be directly compared with that obtained by e.g. the Laser Doppler Imaging (LDI) . The laser Doppler velocimetry and flowmetry are based on blood movement; therefore, the measuring depth will be in the order of $0.5-1 \mathrm{~mm}$ (depending on the tissue type). Thus, the LDI measures the extent of superficial dermal microvascular blood flow and it is suitable e.g. for determining the burn depth [28]. The proposed approach is based on the analysis of heat transfer through the tissues, including also deeper located structures. Thus, evaluation results of the blood perfusion obtained with these two methods may differ since they are estimated for different skin tissue volumes and structures. Moreover, at present the laser Doppler instrument cannot provide absolute perfusion values (measurements are usually expressed as arbitrary perfusion units). To enable comparison of the results the laser Doppler should be appropriately calibrated. On the other hand, taking into consideration 
the assumption of equal perfusion values in deeper skin layers, correlation between our method and other LDI methods could be undertaken in the next step of research.

The uncertainty of measurements did not exceed $30 \%$. This result can be regarded as quite satisfactory taking into account the complexity of skin structure and influence of many factors on quantitative assessment of its parameters.

\section{Acknowledgements}

This work was supported by the National Science Centre Poland (project number DEC2013/11/20 N/ST7/02630).

\section{References}

[1] Pennes, H.H. (1948). Analysis of tissue and arterial blood temperatures in the resting human forearm. Journal of Applied Physiology, 1(2), 93-122.

[2] Chen, M.M., Holmes, K.R. (1980). Microvascular Contributions in Tissue Heat Transfer. Annals of the New York Academy of Sciences, 335(1), 137-150.

[3] Wulff, W. (1974). The Energy Conservation Equation for Living Tissues. IEEE Transactions-Biomedical Engineering, 21(6), 494-495.

[4] Cho, Y.I. (1992). Bioengineering Heat Transfer. Advances in Heat Transfer, Hartnett, J.P., Irvine, T.F. (ed.), Academic Press Inc, San Diego, USA.

[5] Weinbaum, S., Jiji, L.M., Lemons, D.E. (1984) Theory and experiment for the effect of vascular microstructure on surface tissue heat transfer. Part I. Anatomical foundation and model conceptualization. ASME Journal of Biomechanical Engineering, 106(4), 321-330.

[6] Weinbaum, S., Jiji, L.M. (1985). A new simplified bioheat equation for the effect of blood flow on local average tissue temperature. ASME Journal of Biomechanical Engineering, 107(2), 131-139.

[7] Minkowycz, W.J., Sparrow, E.M., Abraham, J.P. (2009). Advances in Numerical Heat Transfer: Vol. 3. CRC Press, Boca Raton, USA.

[8] Zolfaghari, A., Maerefat, M. (2010). A New Simplified Thermoregulatory Bioheat Model for Evaluating Thermal Response of the Human Body to Transient Environmen. Building and Environment, 45(10), 2068-2076

[9] Zolfaghari, A., Maerefat, M. (2011). Bioheat transfer. Developments in heat transfer, Dos Santos Bernardes, M.A. (ed.), InTech.

[10] Jasiński, M. (2008). Modelling of 1D bioheat transfer with perfusion coefficient dependent on tissue necrosis. ScientificResearch of the Institute of Mathematics and Computer Science, Czestochowa University of Technology, 7(1), 57-62.

[11] Khanafer, K., Vafai, K. (2009). Synthesis of mathematical models representing bioheat transport. Advances in Numerical Heat Transfer, CRC Press, New York, Chap. 1, 1-28.

[12] Ng, E.Y.K., Tan, H.M., Ooi, E.H. (2009). Boundary element method with bioheat equation for skin burn injury. Burns, 35(7), 987-997.

[13] Strakowska, M., De Mey, G., Wiecek B., Strzelecki, M. (2015). A three layer model for the thermal impedance of the human skin: modelling and experimental measurements. Journal of Mechanics in Medicine and Biolog, 15(4).

[14] Strakowska, M., Strzelecki, M., Kaszuba, A. (2014). Novel methodology of medical screening using IR thermography. SPA 2014. Signal Processing. Algorithms, Architecture, Arrangements, and Applicatiuons. Conference proceedings, 172-175.

[15] Strakowska, M., Kaszuba, A., Wiecek, B., Strzelecki, M. De Mey, G. (2015). System and software for thermal images screening in medicine - application to psoriasis. Quantitative InfraRed Thermography, 12(2), $127-136$. 
[16] Nowakowski, A., Kaczmarek, M. (2011). Active Dynamic Thermography - Problems of implementation in medical diagnostics. Quantitative InfraRed Thermography Journal, 8(1), 89-106.

[17] Yue, K., Zhang, X., Yu, F. (2004). An Analytic Solution of One-dimensional Steady-state Pennes' Bioheat Transfer Equation in Cylindrical Coordinates. J. of Thermal Science, 13(3), 255-258.

[18] Shih, T.C., Yuan, P., Lin, W.L., Kou, H.S. (2007). Analytical analysis of the Pennes bioheat transfer equation with sinusoidal heat flux condition on skin surface. Medical Engineering \& Physics, 29(9), 946953.

[19] Souza, C.F.L., Souza, M.V.C., Colac, M.J., Caldeira, A.B., Scofano Neto, F. (2014). Inverse determination of blood perfusion coefficient by using different deterministic and heuristic techniques. Journal of the Brazilian Society of Mechanical Sciences and Engineering, 36(1), 193-206.

[20] Strakowska, M, Strakowski, R., Wiecek, B., Strzelecki, M. (2012). Cross-correlation based movement correction method for biomedical dynamic infrared imaging. Proc. of the 11th International Conference on Quantitative InfraRed Thermography, Naples, Italy.

[21] Hooke, R., Jeeves, T.A. (1961). Direct search solution of numerical and statistical problems. Journal of the Association for Computing Machinery (ACM), 8(2), 212-229.

[22] Chinneck, J.W. (2009). Practical Optimization: A Gentle Introduction. Carleton University, Canada.

[23] Gowrishankar, T.R., Stewart, D.A, Gregory, M.T., Weaver, J.C. (2004). Transport lattice models of heat transport in skin with spatially heterogeneous, temperature-dependent perfusion. Biomedical Engineering Online, 3,42 .

[24] Jiang, S.C., Ma, N., Li, H.J., Zhang, X.X. (2002). Effects of thermal properties and geometrical dimensions on skin burn injuries. Burns, 28(8), 713-717.

[25] Evaluation of measurement data - Guide to the expression of uncertainty in measurement, JCGM 100:2008.

[26] Pacholski, K., Wiecek, B. (2015). Practical assessment of accuracy of thermographic indirect measurements. Measurement Automation Monitoring, 61(6), 278-281.

[27] Jakubowska, T., Wiecek, B., Wysocki, M., Drews-Peszynski, C., Strzelecki, M. (2004). Classification of breast thermal images using artificial neural networks. Proc. of 26 th Annual International Conference of the IEEE Engineering in Medicine and Biology Society, 26, 1155-1158

[28] Gill, P. (2013). The critical evaluation of laser Doppler imaging in determining burn depth. International Journal Burns Trauma, 3(2), 72-77. 